\title{
Overview of recent ALICE results
}

\author{
Igor Lakomov (for the ALICE Collaboration) $)^{1, \star}$ \\ ${ }^{1}$ European Organization for Nuclear Research (CERN), CH-1211, Geneva 23, Switzerland
}

\begin{abstract}
ALICE is one of the four largest LHC experiments. It is dedicated to the study of the properties of the deconfined state of matter formed at large energy densities in heavy-ion collisions - the Quark-Gluon Plasma. The ALICE Collaboration also participated in the $\mathrm{pp}$ and $\mathrm{p}-\mathrm{Pb}$ data-taking periods at the LHC. An overview of recent ALICE results is presented for three collision systems: $\mathrm{pp}, \mathrm{p}-\mathrm{Pb}$ and $\mathrm{Pb}-\mathrm{Pb}$.
\end{abstract}

\section{Introduction}

The high energy densities reached in heavy-ion collisions at LHC energies lead to the formation of a deconfined state of matter - the Quark-Gluon Plasma (QGP). The ALICE detector allows one to study the properties of this state at the LHC. Since the lifetime of the QGP is very small it cannot be observed directly. Measurement of the final-state particle yields is one of the strongest tools used for such studies. These yields in heavy-ion collisions are then compared to the expected yields from a superposition of incoherent pp collisions scaled by the number of elementary binary collisions. The ratio of the particle yield in heavy-ion collisions to the corresponding scaled yield in pp collisions is called the Nuclear Modification Factor $\left(R_{\mathrm{AA}}\right)$. In the presence of any hot nuclear matter effects related to the QGP formation, $R_{\mathrm{AA}}$ is not equal to unity. However heavy-ion collisions are more complicated, and there are some Cold Nuclear Matter (CNM) effects playing a role. The latter also might affect the $R_{\mathrm{AA}}$. In order to disentangle hot and CNM effects in heavy-ion collisions, the study of pA collisions is necessary. In pA collisions the energy densities are lower than in nucleus-nucleus collisions, and the formation of the QGP is much less probable. Thus, pA collisions allow one to study pure CNM effects. It is also usually done in terms of the Nuclear Modification Factor, which for $\mathrm{pA}$ collisions is referred to as $R_{\mathrm{pA}}$. In addition to global properties of the QGP, the geometry of the collision system can be studied using other observables, like elliptic flow. For a more detailed description of the system, differential measurements in transverse momentum $\left(p_{\mathrm{T}}\right)$, rapidity $(y)$ and centrality are usually performed. This article presents an overview of the latest ALICE results for different collision systems.

\section{Charged particle and open HF production}

ALICE recently published one of its first results for $\mathrm{Pb}-\mathrm{Pb}$ collisions at $\sqrt{s_{\mathrm{NN}}}=5.02 \mathrm{TeV}$, the pseudorapidity density of charged particles [1]. The left panel of Fig. 1 shows its dependence on the

^e-mail: igor.lakomov@cern.ch 
collision energy compared to other experimental measurements. The rising trends established by the low-energy data for minimum bias pp, $\mathrm{p}(\mathrm{d})-\mathrm{A}$ collisions as well as for central AA collisions are confirmed by the ALICE measurements. Moreover, the fact that $\mathrm{p}-\mathrm{Pb}$ and $\mathrm{d}-\mathrm{Au}$ measurements lay on the curve for $\mathrm{pp}$ collisions leads to the conclusion that the strong rise seen in nucleus-nucleus collisions is not solely related to the multiple interactions undergone by the participants since the proton in $\mathrm{pA}$ collisions also encounters multiple nucleons in the nucleus. In the right plot of Fig. 1 the centrality dependence of the pseudorapidity density of charged particles is shown. The results for $\mathrm{Pb}-\mathrm{Pb}$ at 5.02 $\mathrm{TeV}$ are compared to those from $\mathrm{pp}$ and $\mathrm{Pb}-\mathrm{Pb}$ both at $2.76 \mathrm{TeV}$ and to $\mathrm{p}-\mathrm{Pb}$ measurements at 5.02 $\mathrm{TeV}$. A strong dependence on the average number of participants $N_{\text {part }}$ is seen: $\left\langle\frac{d N}{d \eta}\right\rangle /\left(\left\langle N_{\text {part }}\right\rangle / 2\right)$ is increasing with the increase of $N_{\text {part }}$, i.e. from peripheral to central collisions. The data clearly show the scaling of the centrality dependence with energy and collision system . In order to quantify the
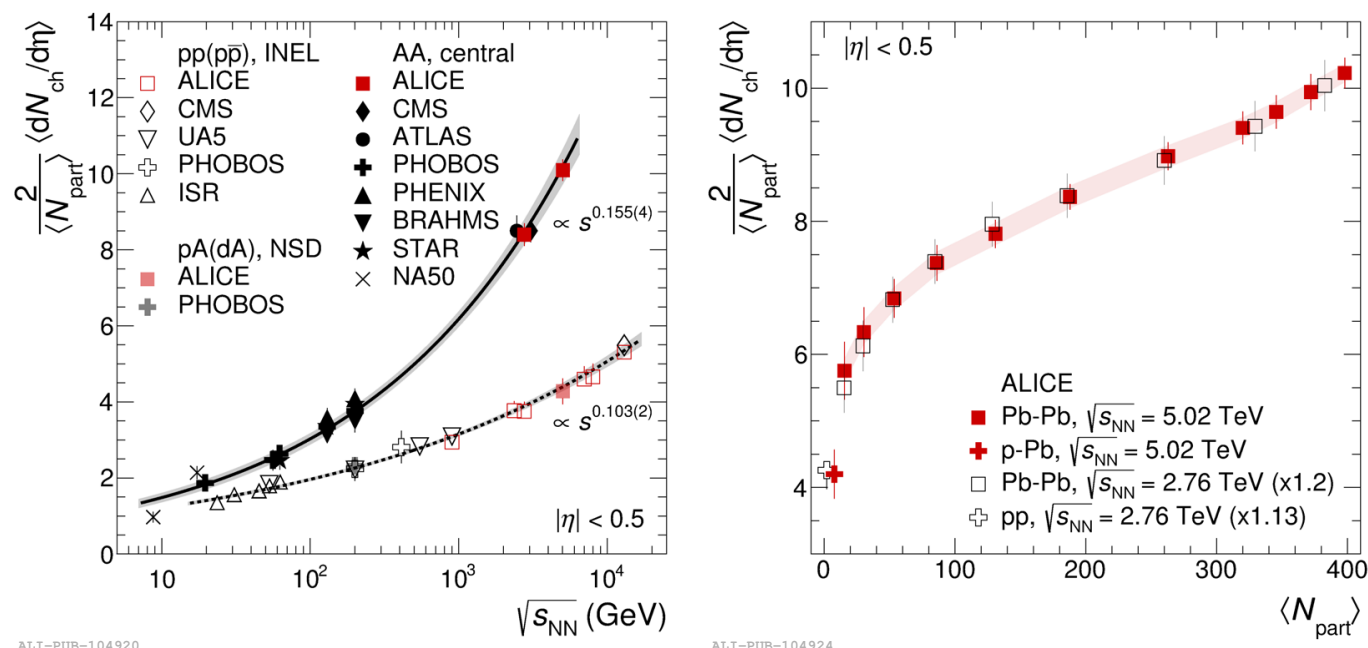

Figure 1: Pseudorapidity density of charged particles $\left\langle\frac{d N}{d \eta}\right\rangle /\left(\left\langle N_{\text {part }}\right\rangle / 2\right)$ as a function of average number of participants $\left(\left\langle N_{\text {part }}\right\rangle\right)$ for central (left) and $0-80 \%$ central (right) collisions. In the left panel the results from other experiments for various collision systems and energies are also shown. In the right panel $\mathrm{pp}$ and $\mathrm{Pb}-\mathrm{Pb}$ results are scaled by 1.13 and 1.2 , respectively.

nuclear matter effects in $\mathrm{p}-\mathrm{Pb}$ and $\mathrm{Pb}-\mathrm{Pb}$ collisions ALICE measured the nuclear modification factors for identified particles. In the left panel of Fig. 2 the $R_{\mathrm{pPb}}$ measurements are presented for charged hadrons, pions, kaons and protons [2]. At high $p_{\mathrm{T}}, R_{\mathrm{pPb}}$ is consistent with unity for all species, while at intermediate $p_{\mathrm{T}}$, in the Cronin effect region, a mass ordering is seen with an indication of proton enhancement. The right panel of Fig. 2 shows the $R_{\mathrm{PbPb}}$ for D-mesons which is compared to previous $R_{\mathrm{pPb}}$ measurements for D-mesons [3]. For both central and semi-central $\mathrm{Pb}-\mathrm{Pb}$ collisions $\mathrm{D}$-meson production is suppressed, up to a factor of 5-6 and 3, respectively, at $p_{\mathrm{T}} \sim 10 \mathrm{GeV} / c$. Results for $\mathrm{p}-\mathrm{Pb}$ collisions in both panels of Fig. 2 are indicative of the hot nuclear matter origin of the corresponding suppression seen in $\mathrm{Pb}-\mathrm{Pb}$ collisions. 

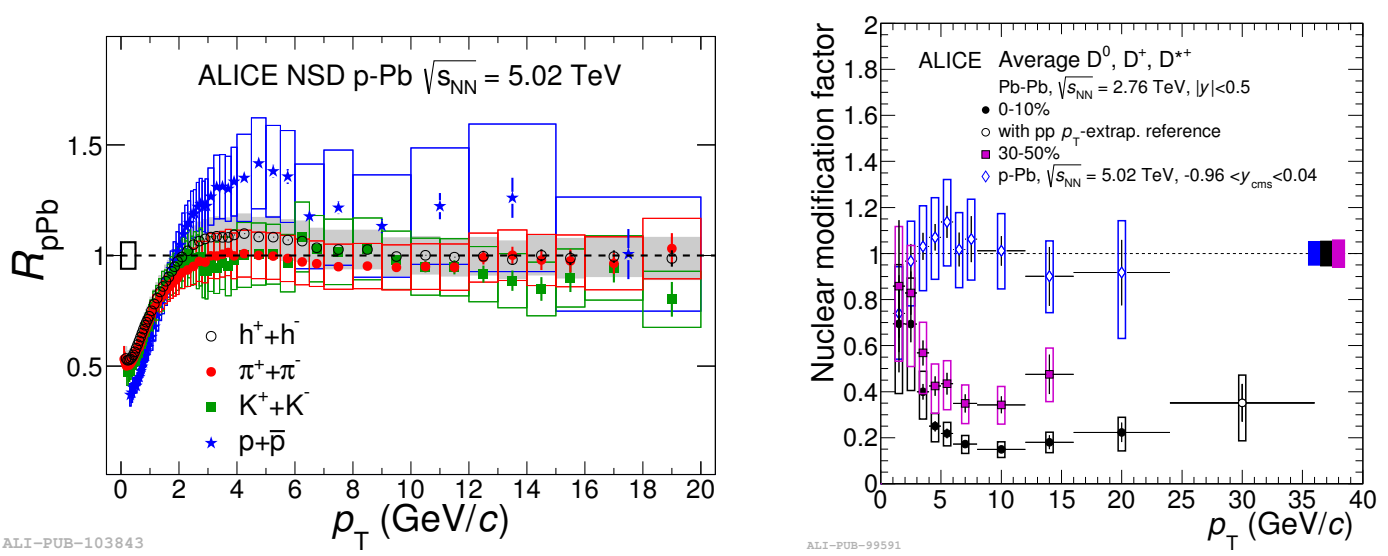

Figure 2: Nuclear modification factors of charged particles in $\mathrm{p}-\mathrm{Pb}$ collisions (left), and of D-mesons in $\mathrm{Pb}-\mathrm{Pb}$ collisions (right). In the left panel results are shown separately for different particle species: charged hadrons, pions, kaons and protons. In the right panel the results are shown for central $(0-$ $10 \%)$ and semi-central (30-50\%) $\mathrm{Pb}-\mathrm{Pb}$ collisions, and compared to the ALICE $\mathrm{p}-\mathrm{Pb}$ measurements.

\section{Jets and flow}

For a deeper understanding of the initial collision geometry, transport mechanisms etc., the momentum anisotropy of the final state particles is usually studied. This is done experimentally in terms of the anisotropic flow coefficients representing the coefficients of the Fourier decomposition of the particle angular distribution. ALICE measured $v_{2}, v_{3}$ and $v_{4}$ of the charged particles angular distribution in $\mathrm{Pb}-\mathrm{Pb}$ collisions at $5.02 \mathrm{TeV}$ and compared them to the corresponding results at lower energy $(2.76$ $\mathrm{TeV}$ ) [4]. As can be seen from the left panel of Fig. 3, the values of $v_{2}, v_{3}$ and $v_{4}$ increase with $\sqrt{s_{\mathrm{NN}}}$ from 2.76 to $5.02 \mathrm{TeV}$. In the $0-50 \%$ centrality range they increase by $(3.0 \pm 0.6) \%,(4.3 \pm 1.4) \%$ and $(10.2 \pm 3.8) \%$, respectively. Moreover, this increase seems to be independent of centrality as seen from the corresponding ratios. Hydrodynamical models describe these results fairly well. In the right top and bottom panels of Fig. 3, the ALICE $v_{2}$ measurements for charged jets in central and semi-central $\mathrm{Pb}-\mathrm{Pb}$ collisions at $2.76 \mathrm{TeV}$, respectively, are shown [5]. With a significance higher than $3 \sigma$ the semi-central $\mathrm{Pb}-\mathrm{Pb}$ measurements indicate a non-zero $v_{2}^{\text {ch jet }}$. The observed behavior indicates a pathlength dependence of the in-medium parton energy loss. Larger uncertainties on the measurements for central $\mathrm{Pb}-\mathrm{Pb}$ collisions preclude drawing a strong conclusion on the magnitude of $v_{2}^{\text {ch jet }}$. Moreover, for central $\mathrm{Pb}-\mathrm{Pb}$ collisions, the fluctuations in the initial state may contribute to the measured nonzero $v_{2}^{\text {ch jet }}$. The $v_{2}^{\text {ch jet }}$ results are compared to single particle $v_{2}$ from ALICE and CMS and $v_{2}^{\text {calo jet }}$ measured by ATLAS. While they cannot be directly quantitatively compared given the difference in the energy scale, the results agree fairly well showing a positive $v_{2}$ for both charged particles and jets to high $p_{\mathrm{T}}$ in central and semi-central $\mathrm{Pb}-\mathrm{Pb}$ collisions. This observation indicates that the sensitivity to the collision geometry persists up to high $p_{\mathrm{T}}$.

\section{Quarkonium production}

The study of the production of quarkonia, bound states of a charm (beauty) quark and its antiquark, is of particular interest. Heavy and tightly bound, the heavy quark pairs are produced in the initial hard 

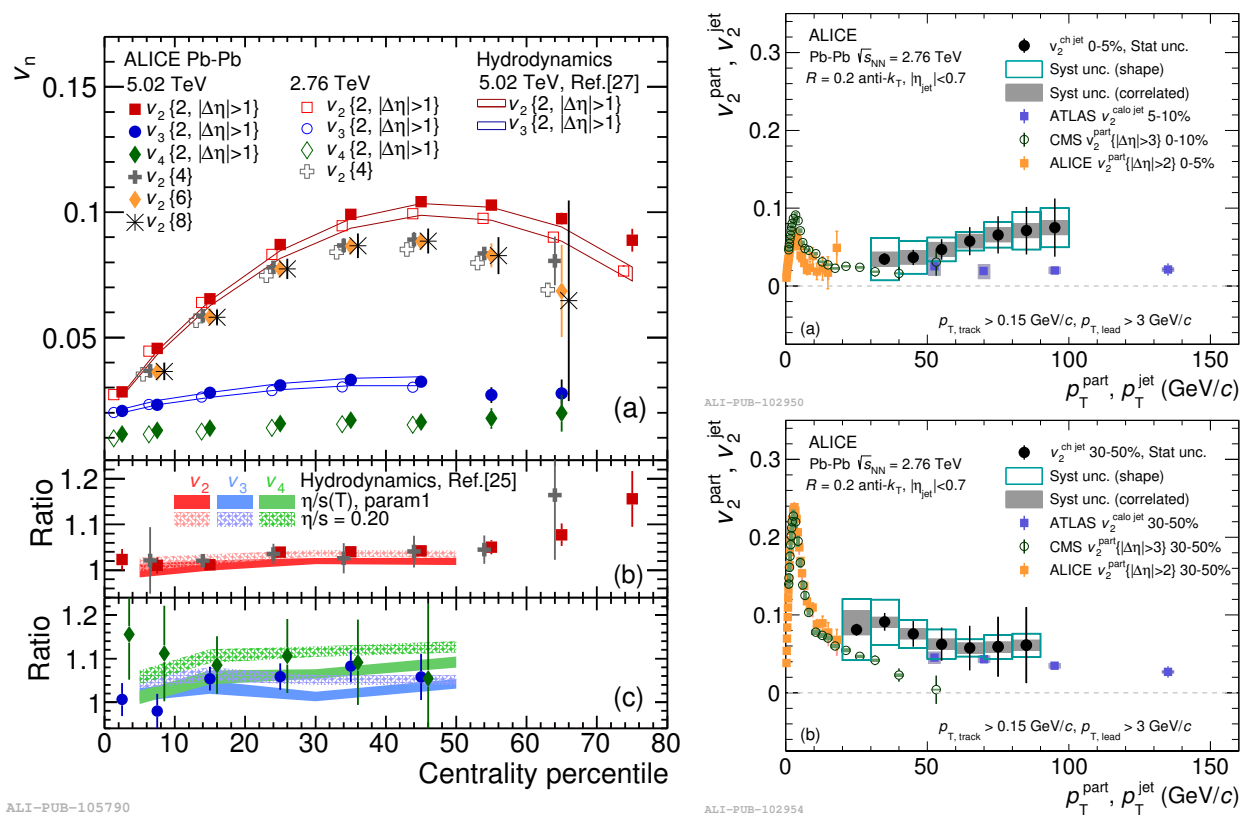

Figure 3: Measurements of the anisotropic flow of charged hadrons (left) and of charged jets (right) in $\mathrm{Pb}-\mathrm{Pb}$ collisions. In the left panel ALICE results for 5.02 and $2.76 \mathrm{TeV}$ as a function of centrality are compared. In the right part of the figure, results are shown for central $(0-5 \%$, top $)$ and semicentral $\left(30-50 \%\right.$, bottom) collisions as a function of $p_{\mathrm{T}}$. In both plots on the right, the corresponding measurements from ATLAS and CMS are also shown.

partonic collisions. Sequential suppression of quarkonium states with the increase of the temperature of the collision may be used as a QGP thermometer [6,7], therefore thorough study of quarkonium production is crucial for understanding the physics in heavy-ion collisions. Figure 4 shows the ALICE measurements of the $\mathrm{J} / \psi$ suppression in $\mathrm{Pb}-\mathrm{Pb}$ collisions at $2.76 \mathrm{TeV}$ as a function of centrality [8] (left panel) and of $p_{\mathrm{T}}$ [9] (right panel). The $\mathrm{J} / \psi$ production is suppressed in $\mathrm{Pb}-\mathrm{Pb}$ collisions. The suppression increases with increasing centrality and for higher $p_{\mathrm{T}}$. A comparison to the results from PHENIX for $\mathrm{Au}-\mathrm{Au}$ collisions at $0.2 \mathrm{TeV}$ in the left panel of Fig. 4 indicates a larger $\mathrm{J} / \psi$ suppression at RHIC energies compared to that seen at about 10 times higher collision energies at the LHC. This is the effect of recombination which is expected to be significant at LHC energies while at RHIC energies it is almost negligible. At low $p_{\mathrm{T}}$ its effect is expected to be higher, thus high- $p_{\mathrm{T}} \mathrm{J} / \psi$ are more suppressed. However it was not expected to explain the strong low- $p_{\mathrm{T}} \mathrm{J} / \psi$ excess at low $N_{\text {part }}$ (corresponding to peripheral collisions) clearly seen in the right panel of Fig. 4. After more detailed study this excess was understood as an effect from coherent photo-production of $\mathrm{J} / \psi$ in ultra-peripheral collisions [9]. After the extensive analysis of the $\mathrm{J} / \psi$ production in $\mathrm{p}-\mathrm{Pb}$ collisions where the $\mathrm{CNM}$ effects were quantified as a function of $p_{\mathrm{T}}$, rapidity and centrality [10-12], and the corresponding measurement of the $\psi(2 \mathrm{~S})$ production in $\mathrm{p}-\mathrm{Pb}$ collisions integrated over centrality [13], ALICE recently published the results on the $\psi(2 \mathrm{~S})$ production in $\mathrm{p}-\mathrm{Pb}$ collisions as a function of centrality [14]. Figure 5 shows the double ratio of the $\psi(2 \mathrm{~S})$ and $\mathrm{J} / \psi$ cross sections in $\mathrm{p}-\mathrm{Pb}$ collisions with respect to pp collisions: $\left[\sigma_{\psi(2 \mathrm{~S})} / \sigma_{\mathrm{J} / \psi}\right]_{\mathrm{pPb}} /\left[\sigma_{\psi(2 \mathrm{~S})} / \sigma_{\mathrm{J} / \psi}\right]_{\mathrm{pp}}$ as a function of the number of binary collisions 

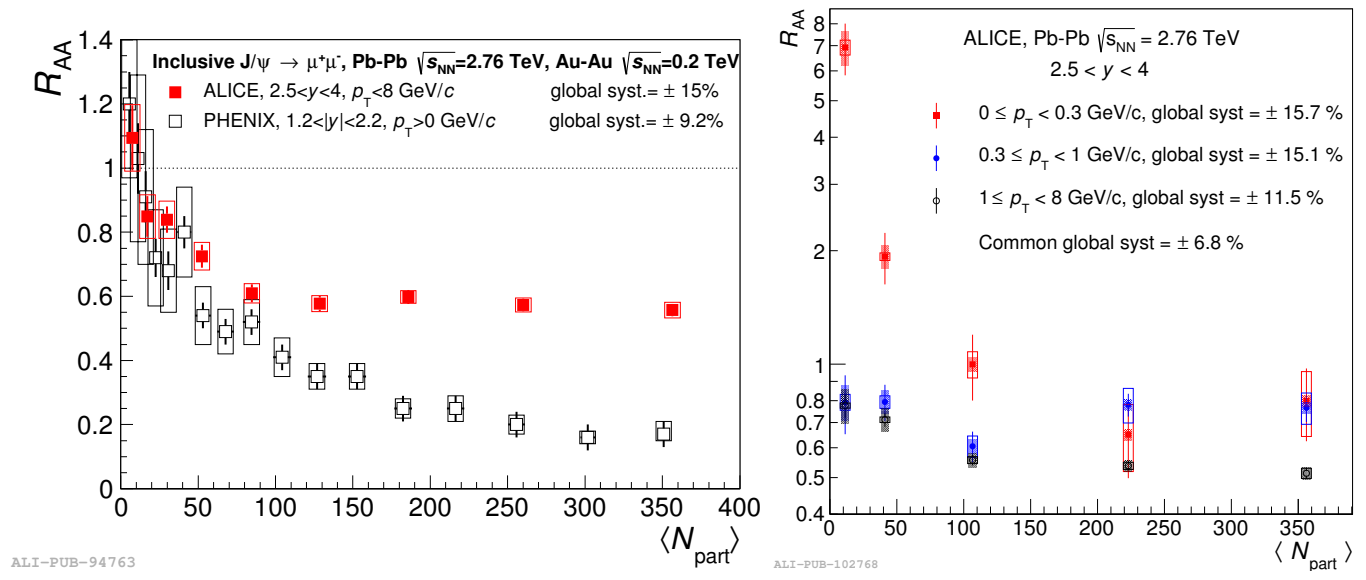

Figure 4: Nuclear modification factor for $\mathrm{J} / \psi$ in $\mathrm{Pb}-\mathrm{Pb}$ collisions as a function of $N_{\text {part }}$ integrated over $p_{\mathrm{T}}$ (left) and in different $p_{\mathrm{T}}$ intervals (right). In the left panel the ALICE data are compared to the corresponding results from PHENIX for $\mathrm{Au}-\mathrm{Au}$ collisions at $0.2 \mathrm{TeV}$.

$N_{\text {coll }}$ (left panel) and of the crossing time $\left\langle\tau_{\mathrm{c}}\right\rangle$ of the $c \bar{c}$ pair in the nuclear matter (right panel). The measured double ratio indicates a decreasing trend with increasing centrality of the collisions (higher $N_{\text {coll }}$ values) for forward and backward rapidity intervals, which agrees fairly well with theoretical model calculations including final-state effects. However large uncertainties for the forward- $y$ measurements are also consistent with no centrality dependence. Surprisingly, the ALICE measurements show similar behaviour to the PHENIX d-Au results in the mid-y interval and with about 25 times lower collision energy. The crossing time was varied by studying different $p_{\mathrm{T}}$ and centrality intervals. At forward rapidity, the $\tau_{\mathrm{c}}$ values are much smaller than the resonance formation time $\tau_{\mathrm{f}}$, excluding a significant role of final-state interactions with nuclear matter. Effects occurring at later times, such as the break-up by co-movers in the hadronic gas, are suitable candidates for the explanation of the observed $\psi(2 \mathrm{~S})$ suppression. Significantly larger values of $\tau_{\mathrm{c}}$ at backward rapidity are still smaller than $\tau_{\mathrm{f}}$. The observed $\tau_{\mathrm{c}}$-scaling of the double ratios might indicate an effect at least partly related to a dissociation of the fully-formed resonance in nuclear matter.

\section{Conclusions}

Some recent ALICE results are presented in this overview for different collision systems ( $\mathrm{pp}, \mathrm{p}-\mathrm{Pb}$, $\mathrm{Pb}-\mathrm{Pb}$ ), including both measurements from Run I and Run II of the data-taking period at the LHC. Comparisons to theoretical models and results from other experiments lead to a deeper understanding of the physics of high-energy particle collisions and give additional constraints to theoreticians to tune their models. Many other ALICE results will appear soon based on the collected and coming data.

\section{References}

[1] J. Adam et al. (ALICE), Phys. Rev. Lett. 116, 222302 (2016), 1512.06104

[2] J. Adam et al. (ALICE) (2016), 1601.03658

[3] J. Adam et al. (ALICE), JHEP 03, 081 (2016), 1509. 06888 

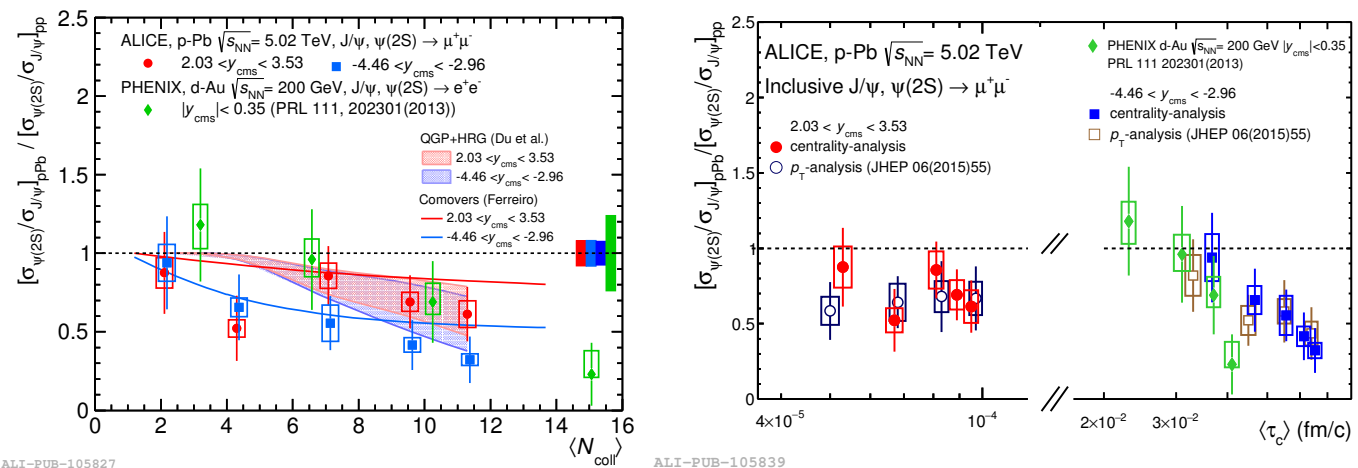

Figure 5: Double ratio of the $\psi(2 \mathrm{~S})$ and $\mathrm{J} / \psi$ cross sections in $\mathrm{p}-\mathrm{Pb}$ collisions with respect to $\mathrm{pp}$ collisions: $\left[\sigma_{\psi(2 \mathrm{~S})} / \sigma_{\mathrm{J} / \psi}\right]_{\mathrm{pPb}} /\left[\sigma_{\psi(2 \mathrm{~S})} / \sigma_{\mathrm{J} / \psi}\right]_{\mathrm{pp}}$ as a function of the number of binary collisions $N_{\text {coll }}$ (left panel) and of the crossing time $\left\langle\tau_{c}\right\rangle$ (right panel) of the $c \bar{c}$ pair in the nuclear matter. ALICE results are also compared to the PHENIX measurements for $\mathrm{d}-\mathrm{Au}$ collisions at $0.2 \mathrm{TeV}$.

[4] J. Adam et al. (ALICE), Phys. Rev. Lett. 116, 132302 (2016), 1602.01119

[5] J. Adam et al. (ALICE), Phys. Lett. B753, 511 (2016), 1509.07334

[6] T. Matsui, H. Satz, Phys. Lett. B178, 416 (1986)

[7] H. Satz (2006), hep-ph/0602245

[8] J. Adam et al. (ALICE), JHEP 05, 179 (2016), 1506.08804

[9] J. Adam et al. (ALICE), Phys. Rev. Lett. 116, 222301 (2016), 1509.08802

[10] B.B. Abelev et al. (ALICE), JHEP 02, 073 (2014), 1308.6726

[11] J. Adam et al. (ALICE), JHEP 06, 055 (2015), 1503.07179

[12] J. Adam et al. (ALICE), JHEP 11, 127 (2015), 1506.08808

[13] B.B. Abelev et al. (ALICE), JHEP 12, 073 (2014), 1405 . 3796

[14] J. Adam et al. (ALICE), JHEP 06, 050 (2016), 1603.02816 